\title{
Central obesity and smoking are key modifiable risk factors for elevated C-reactive protein in Asian individuals who are not eligible for statin therapy
}

\author{
$\mathrm{CM} \mathrm{Khoo}^{1}, \mathrm{M} \mathrm{Tan}^{2}, \mathrm{Y} \mathrm{Wu}{ }^{2}, \mathrm{CH} \mathrm{Wai}{ }^{3}, \mathrm{~T} \mathrm{Subramaniam}^{4}, \mathrm{~J} \mathrm{Lee}^{1,2}$ and ES Tai ${ }^{1,2,5}$ \\ ${ }^{1}$ Department of Medicine, Yong Loo Lin School of Medicine, National University of Singapore, Singapore; ${ }^{2}$ Department of \\ Epidemiology and Public Health, Yong Loo Lin School of Medicine, National University of Singapore, Singapore; ${ }^{3}$ Department \\ of Endocrinology, Singapore General Hospital, Singapore; ${ }^{4}$ Department of Medicine, Khoo Teck Puat Hospital, Singapore and \\ ${ }^{5}$ Duke-National University of Singapore Graduate Medical School, Singapore
}

\begin{abstract}
Objective: Statin therapy reduces coronary heart disease (CHD) and mortality in individuals with elevated C-reactive protein (CRP) but low-density lipoprotein cholesterol below the threshold at which statin therapy is recommended. We determined the proportion of individuals with elevated CRP in whom statin therapy was not indicated, and examined predictors for elevated CRP in a multi-ethnic Asian population.

Design: We studied 3404 participants (Chinese, Malays and Asian-Indians) without a history of hypercholesterolemia living in Singapore (mean age \pm s.d.: $48.9 \pm 11.2$ years). Eligibility for statin therapy was determined based on the National Cholesterol Education Program (NCEP) Expert Panel on Detection, Evaluation and Treatment of High Blood Cholesterol in Adults (Adult Treatment Panel III (ATPIII)) guideline. CRP was measured by high-sensitivity enzyme-linked immunosorbent assay method. CRP level greater than $2 \mathrm{mg} \mathrm{I}^{-1}$ was considered as elevated.

Results: Elevated CRP was found in $29.3 \%$ participants who were not eligible for statin therapy $(n=2974)$. Elevated CRP was more common in females and amongst those of Malay or Asian-Indian ethnicity. Compared with participants with low CRP, those with elevated CRP were shown to have higher levels of obesity, blood pressure, triglyceride and insulin resistance (IR), but lower high-density lipoprotein cholesterol levels (all $P_{s}<0.001$ ). After multivariate analysis, gender (odds ratio (OR) 3.34 for females), ethnicity (Malay OR 1.57, 95\% confidence interval (Cl) 1.25-1.96; Asian-Indian OR 1.97, 95\% Cl 1.55-2.50), waist circumference (OR 1.06, 95\% Cl 1.05-1.07), smoking (OR 1.49, 95\% Cl 1.08-2.05) and IR (OR 1.14, 95\% Cl 1.07-1.22) were significant predictors of CRP (all Ps-values $<0.05$ ).

Conclusion: Routine measurement of CRP identifies a substantial number of Asian individuals at risk of CHD in whom statin therapy is not currently indicated, particularly in women and certain ethnic groups (Malays and Asian Indians). Weight loss and smoking cessation are important measures to reduce the proportion of individuals with elevated CRP.

Nutrition and Diabetes (2011) 1, e8; doi:10.1038/nutd.2011.4; published online 23 May 2011
\end{abstract}

Keywords: C-reactive protein; statin; NCEP-ATPIII; Asian; obesity

\section{Introduction}

The National Cholesterol Education Program (NCEP) Expert Panel on Detection, Evaluation and Treatment of High Blood Cholesterol in Adults (Adult Treatment Panel III (ATPIII)) guideline has recommended that patients should be treated with lipid-lowering therapy if the level of low-density

Correspondence: Dr ES Tai, Department of Medicine, Yong Loo Lin School of Medicine, National University of Singapore, 16 Medical Drive, 117597, Singapore.

E-mail: eshyong@pacific.net.sg

Received 3 January 2011; accepted 11 April 2011 lipoprotein cholesterol exceeds a specific threshold. To optimize the cost effectiveness and minimize the risk of lipid-lowering therapy, these thresholds are calibrated to the estimated risk for the individual patient, such that the most intensive therapy is recommended for those at the highest risk. The NCEP-ATPIII guideline uses the Framingham Risk Score (FRS), which is the most widely validated and used cardiovascular risk assessment tool that helps to risk stratify individuals for future risk of coronary heart disease (CHD). ${ }^{1}$

It is increasingly recognized that atherosclerosis is an inflammatory disease $\mathrm{e}^{2}$ and that $\mathrm{C}$-reactive protein (CRP), as a marker of inflammation, is associated with elevated risk of CHD. ${ }^{3}$ CRP is not included in the FRS that is used in the 
NCEP-ATPIII guideline for risk assessment. Recent studies in both men ${ }^{4}$ and women ${ }^{5}$ have shown that for individuals with the same level of risk assessed using the FRS, individuals with high CRP have elevated risk of CHD. Most recently, the JUPITER trial (Justification for the Use of Statins in Prevention: an Intervention Trial Evaluating Rosuvastatin) showed that, in patients with elevated CRP $\left(>2 \mathrm{mgl}^{-1}\right)$ but low-density-lipoprotein cholesterol (LDL-C) levels below the currently recommended thresholds for initiating lipid lowering therapy, statin therapy reduced the risk of CHD and all-cause mortality LDL. ${ }^{6}$ The increased risk of CHD with increasing levels of CRP is also observed in populations in Asia. However, it should be noted that in several Asian populations, the level of CRP is much lower than in Western populations. ${ }^{7-10}$ In a study conducted in Japan, individuals in the top quartile of CRP had over three times increased risk of $\mathrm{CHD},{ }^{11}$ but the median CRP level in this quartile was only $1.47 \mathrm{mgl}^{-1}$, which was well below the level used as an inclusion criterion in the JUPITER study. It might be suggested that the current definitions of elevated CRP and the findings of the JUPITER study have little relevance to Asian populations.

To better understand these issues, we examined data from a large, population-based study, which included three major ethnic groups (Chinese, Malays and Asian Indians) living in Singapore. We sought to determine the proportion and the characteristics of the population with elevated CRP levels, but LDL-C levels below the currently recommended threshold for statin therapy, as recommended by the NCEP-ATPIII guideline. We also determined the major determinants for elevated CRP in this population, as this may provide information required to implement targeted screening programs and may point toward other targets for non-drug intervention.

\section{Subjects and methods}

\section{Study design and population}

We invited 10747 participants from four population-based, cross-sectional surveys conducted in Singapore (1982-1998) to participate in a repeat examination from 2004 to 2007. The four studies include the Thyroid and Heart Study 1982$1984,{ }^{12}$ the National Health Survey $1992,{ }^{13}$ the National University of Singapore Heart Study $1993-1995^{14}$ and the National Health Survey 1998. ${ }^{15}$ Briefly, all studies included a random sample of individuals from the Singapore population, with disproportionate sampling stratified by ethnicity to increase the number in the minority ethnic groups (Malays and Asian Indians). Participants' deceased at the time of follow-up were excluded $(n=559)$. Also excluded were six participants who had emigrated and 102 who had errors in the records of their National Registry Identity Card. Participants were contacted to obtain an appointment for investigators to administer the questionnaire at the subject's home. Home visits were attempted on three different occasions before a subject was deemed non-contactable $(n=2306)$. Of those participants who could be contacted, $30(0.4 \%)$ refused to participate. All participants were invited to attend a health examination for additional tests and collection of biological specimens shortly after the home visit. A total of 7744 (76.8\% response rate) participants completed the questionnaire; 5164 of them $(66.7 \%$ of those who completed the questionnaire or $51.2 \%$ of all eligible participants) also attended the health examination. During the period from 2 April 2005 to 20 February 2006, because of limited resources, ankle-brachial index was determined only for every alternate Chinese participant but for all participants from other ethnic groups. Data for peripheral vascular disease, one of the CHD risk equivalents, were, therefore, not available for 1050 participants and they were thus excluded from the analyses.

Ethics approval was obtained from two institutional review boards (the National University of Singapore and the Singapore General Hospital). Informed consent was obtained before the study was conducted.

\section{Data collection}

Data on demographic and lifestyle factors (alcohol consumption, smoking), as well as medical history (including physician-diagnosed hypertension, diabetes mellitus and hypercholesterolemia), were collected by using intervieweradministered questionnaires. For the health examination, participants were examined the morning following a 10-h overnight fast. Venous blood was drawn and collected in plain and fluoride oxalate tubes and was stored at $4{ }^{\circ} \mathrm{C}$ for a maximum of $4 \mathrm{~h}$ before processing. All biochemical analyses of blood were carried out at the National University Hospital Referral Laboratory, which is accredited by the College of American Pathologists. Serum total cholesterol, triglyceride and high-density lipoprotein cholesterol were measured by using an automated autoanalyzer (ADVIA 2400; Bayer Diagnostics, Tarrytown, NY, USA). LDL-C levels were calculated using the Friedewald formula. Plasma glucose was assayed with enzymatic methods (ADVIA 2400) by using blood collected in fluoride oxalate tubes. The intra-day and inter-day coefficients of variation for total cholesterol, triglyceride, high-density lipoprotein cholesterol and plasma glucose were $0.80-1.57 \%$ and $0.93-1.15 \%, 0-3.85 \%$ and $1.27-3.40 \%, \quad 0.56-0.65 \%$ and $1.18-2.00 \%, 0-0.93 \%$ and $1.68-1.83 \%$, respectively. Insulin resistance (IR) was assessed by the homeostasis model assessment, where IR $=$ (fasting insulin in $\mathrm{mIUl}^{-1} \times$ fasting glucose in $\left.\mathrm{mmoll}^{-1}\right) / 22.5$. CRP was measured using a high-sensitivity enzyme-linked immunosorbent assay method implemented on the COBAS Integra 400 from Roche Diagnostics (Rotkreuz, Switzerland). The intra-assay precision was $0.6-1.3 \%$, whereas the interassay precision was $2.3-3.1 \%$. We defined elevated CRP as $>2.0 \mathrm{mgl}^{-1}$, similar to the criteria that was used in JUPITER trial. ${ }^{6}$ In addition, Jiang et al. ${ }^{16}$ found that a CRP level of $2 \mathrm{mgl}^{-1}$ or more effectively predicted the risk of CHD in the Chinese population. 
Height was measured, without the subject wearing shoes, using a wall-mounted stadiometer. Weight was measured in light clothing using the same digital scale (SECA, model 7822321009; Vogel \& Halke, Hamburg, Germany) for all participants. Body mass index was calculated as weight (in kilogram) divided by height (in meter) squared. An automated blood pressure monitor (Dinamap Pro100V2; Criticon, Norderstedt, Germany) was used to take two blood pressure readings from participants after $5 \mathrm{~min}$ of rest. A third reading was performed if the difference between the two readings of systolic blood pressure was greater than $10 \mathrm{~mm} \mathrm{Hg}$ or of diastolic blood pressure was greater than $5 \mathrm{~mm} \mathrm{Hg}$. Mean values of the closest two readings were calculated. Ankle pressures were measured using a standardized doppler ultrasonic device $(8 \mathrm{MHz}$; SmartdopTM 20EX, Bi-directional blood flow detector, Hadeco, Japan) after a 5-min rest in the supine position. Ankle-brachial index was calculated as the ratio of the higher of the two systolic pressures (from posterior tibial and dorsalis pedis) at the ankle to the average of the right and left brachial artery pressures.

\section{Definitions}

Diabetes mellitus was defined as fasting plasma glucose $\geqslant 7.0 \mathrm{mmoll}^{-1}$ or a known history of diabetes mellitus and currently taking anti-diabetic medications. Hypertension was defined as a systolic blood pressure greater than $140 \mathrm{mmHg}$ or a diastolic blood pressure greater than $90 \mathrm{~mm} \mathrm{Hg}$ or a history of hypertension or current use of antihypertensive medications. Cigarette smoking was defined as individuals who were currently smoking. Peripheral arterial disease was defined as an ankle-brachial index $<0.9$. Each individual was further categorized according to the five criteria for metabolic syndrome based on the International Diabetes Federation criteria: ${ }^{17}$ triglyceride $>1.7 \mathrm{mmoll}^{-1}$; high-density lipoprotein cholesterol $<1.0 \mathrm{mmoll}^{-1}$ in men or $<1.3 \mathrm{mmoll}^{-1}$ in women; blood pressure $>130 / 85 \mathrm{~mm} \mathrm{Hg}$ or known treatment for hypertension; fasting plasma glucose $>5.6 \mathrm{mmoll}^{-1}$ or known treatment for diabetes; and waist circumference $>80 \mathrm{~cm}$ in women and $>90 \mathrm{~cm}$ in men. Individuals showed central obesity, and at least two other risk factors were classified as metabolic syndrome.

\section{Statistical analysis}

We estimated the 10-year risk of CHD for all participants using the FRS. ${ }^{1}$ Participants were assigned to one of the four risk categories according to the NCEP-ATPIII guideline to determine eligibility for cholesterol-lowering treatment, namely a statin therapy. ${ }^{18}$ These categories are (1) low CHD risk: 0-1 risk factor, (2) intermediate low CHD risk: $2+$ risk factors and 10-year risk $<10 \%$, (3) intermediate high CHD risk: $2+$ risk factors and 10-year risk 10-20\% and (4) high CHD risk: CHD risk equivalent with 10-year risk $>20 \%$ (Figure 1). Participants who required statin therapy were those with the LDL-C levels above group-specific thresholds (i.e., LDL-C of 190, 160, 130 and $130 \mathrm{mg} \mathrm{dl}^{-1}$ for risk category group 1 through 4, respectively). Participants who did not meet the NCEP-ATPIII guideline for initiation of statin therapy (i.e., not eligible for statin therapy) were further subdivided into two groups based on CRP cutoff level of $2 \mathrm{mg} \mathrm{l}^{-1}$. Serum CRP levels were limited to maximum of $10 \mathrm{mgl}^{-1}$ as per the American Heart Association/Centers for Disease Control guideline, which states that a level of $>10 \mathrm{mgl}^{-1}$ may indicate an acute inflammatory state or inflammatory disease. ${ }^{19}$

For continuous variables, one-way analysis of variance was used to test the differences between participants with low CRP $\left(\leqslant 2 \mathrm{mgl}^{-1}\right)$ versus high CRP $\left(>2.0 \mathrm{mgl}^{-1}\right)$ levels. The $\chi^{2}$-test was used to compare proportions of categorical variables between the groups. Odds ratios (ORs) and 95\% confidence intervals (CIs) were obtained from a logistic regression model, which examined predictors for CRP in participants who were not eligible for cholesterol-lowering therapy. All data analyses were conducted using Stata 10 for Windows (Stata Corporation, College Station, TX, USA). All values are given as mean (standard deviation) unless stated otherwise. All statistical tests were two-sided, with a statistical significance defined as $P<0.05$.

\section{Results}

For this study, we excluded participants who were on statin treatment $(n=395)$, had missing data on history of heart disease, stroke, hypertension, diabetes mellitus and hypercholesterolemia, BMI and smoking $(n=315)$. Thus, data on

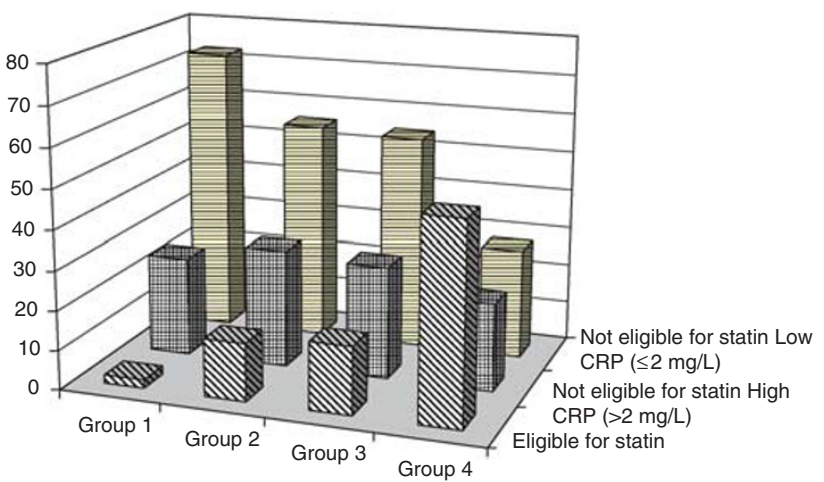

Figure 1 Proportions of participants (\%) who were eligible and not eligible for statin therapy, based on CHD risk categories as recommended by the NCEP-ATPIII guideline. The participants who were not eligible for statin therapy were further divided into low CRP $\left(\leqslant 2 \mathrm{mgl}^{-1}\right)$ and high CRP $\left(>2 \mathrm{mgl}^{-1}\right)$ group. Group 1 -low CHD risk (0-1 risk factor), $n=1946$. Group 2 -intermediate low CHD risk (2+ risk factors and Framingham 10 year risk $<10 \%), n=619$. Group 3 -intermediate high CHD risk $(2+$ risk factors and Framingham 10-year risk 10-20\%), $n=353$. Group 4-high CHD risk (CHD risk equivalent with 10-year risk $>20 \%$ ), $n=486$. 
3404 participants with complete blood and CRP $\left(<10 \mathrm{mg} \mathrm{l}^{-1}\right)$ measurements were used in the analysis.

Overall, 1946 (57.2\%) participants had low CHD risk, 619 (18.2\%) had intermediate low CHD risk, 353 (10.4\%) intermediate high CHD risk and 486 (14.2\%) had high CHD risk. Approximately $12.6 \%$ would qualify for statin therapy, based on the NCEP-ATPIII guideline. The proportion of those in whom a statin was indicated increased as the risk level increased, reaching $56.3 \%$ in those who were at high risk of CHD (Figure 1). A further 29.3\% had elevated CRP, even though statin therapy was not indicated, with a similar proportion across the various risk categories. The majority (73.2\%) of individuals at low risk also had normal CRP levels.

In Table 1, we compared the characteristics of participants with and without elevated CRP among those who were not eligible for statin therapy $(n=2974)$. The characteristics of those who did qualify for statin therapy were presented alongside for comparison. Among those not eligible for statin therapy, individuals with elevated CRP levels were more likely to be older, female and of Malay or Asian-Indian ethnicity. Compared with those with CRP $\leqslant 2 \mathrm{mgl}^{-1}$, participants with CRP $>2 \mathrm{mgl}^{-1}$ were more likely to have hypertension and diabetes mellitus and also had higher blood pressure, obesity (measured by body mass index or waist circumference), plasma triglyceride, LDL-cholesterol and IR (all $P$-values $<0.001$ ). High-density lipoprotein cholesterol was lower in those with elevated CRP levels $(P<0.001)$.
As many of the features associated with elevated CRP were components of the metabolic syndrome, we also determined the number of metabolic risk factors according to the International Diabetes Federation definition of metabolic syndrome in those with and without elevated CRP (Figure 2).

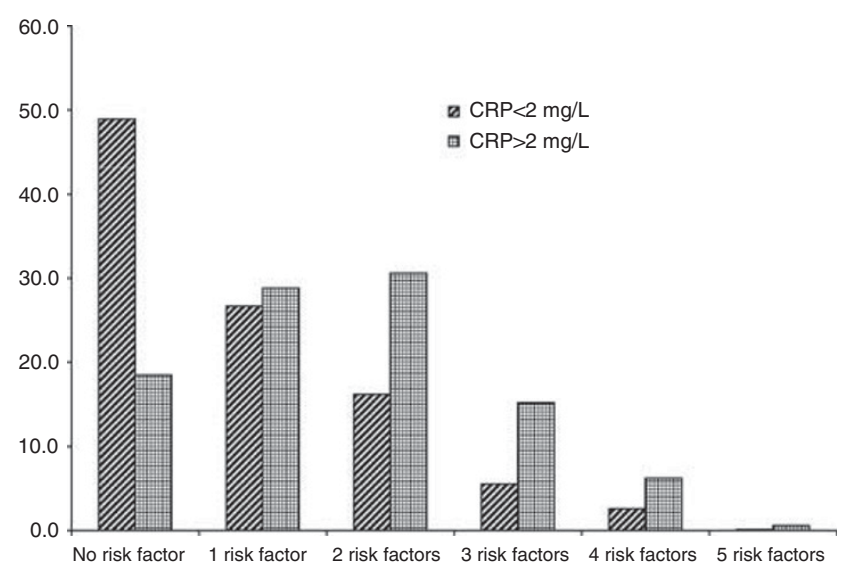

Figure 2 Proportion of participants (\%) with varying numbers of cardiometabolic risk factors in those with and without elevated C-reactive protein (CRP). The participants were divided into low CRP $\left(\leqslant 2 \mathrm{mgl}^{-1}\right)$ and high CRP $\left(>2 \mathrm{mgl}^{-1}\right)$ group. The cardio-metabolic risk factors include triglyceride $>1.7 \mathrm{mmoll}^{-1}$, high-density lipoprotein cholesterol $<1.0 \mathrm{mmol} \mathrm{I}^{-1}$ in men or $<1.3 \mathrm{mmoll}^{-1}$ in women, blood pressure $>130 / 85 \mathrm{~mm} \mathrm{Hg}$ or known treatment for hypertension, fasting plasma glucose $>5.6 \mathrm{mmoll}^{-1}$ or known treatment for diabetes and waist circumference $>80 \mathrm{~cm}$ in women and $>90 \mathrm{~cm}$ in men.

Table 1 Characteristics of participants who were eligible and were not eligible for statin therapy, based on the NCEP-ATPIII guideline

\begin{tabular}{|c|c|c|c|c|}
\hline & \multicolumn{3}{|c|}{ Participants not eligible for statin therapy } & \multirow{2}{*}{$\begin{array}{l}\text { Participants eligible } \\
\text { for statin therapy }\end{array}$} \\
\hline & $\operatorname{Low} C R P\left(\leqslant 2 \mathrm{mgl}^{-1}\right)$ & High $C R P\left(>2 \mathrm{mgl}^{-1}\right)$ & P-value* & \\
\hline$N$ & 2104 & 870 & & 430 \\
\hline Female (\%) & 52.1 & 61.2 & $<0.001$ & 35.4 \\
\hline Ethnicity (\%) & & & $<0.001$ & \\
\hline Chinese & 78.8 & 21.2 & & 39.8 \\
\hline Malay & 61.1 & 38.9 & & 35.4 \\
\hline Indian & 50.3 & 49.7 & & 24.9 \\
\hline Current smoking ${ }^{\mathrm{a}}(\%)$ & 9.5 & 8.9 & 0.576 & 28.8 \\
\hline History of hypertension (\%) & 13.2 & 21.5 & $<0.001$ & 27.0 \\
\hline Diabetes mellitus (\%) & 4.7 & 9.7 & $<0.001$ & 31.0 \\
\hline Age, year & $47.4(11.0)$ & $49.6(10.7)$ & $<0.001$ & $54.9(11.1)$ \\
\hline Body mass index $\left(\mathrm{kg} \mathrm{m}^{-2}\right)$ & $22.7(3.5)$ & $26.0(4.2)$ & $<0.001$ & $25.1(4.0)$ \\
\hline Waist circumference $(\mathrm{cm})$ & $80.6(11.3)$ & $88.4(10.7)$ & $<0.001$ & $89.4(11.1)$ \\
\hline Systolic BP (mm Hg) & $127.5(18.8)$ & $133.6(19.9)$ & $<0.001$ & $142.9(22.9)$ \\
\hline Diastolic BP (mm Hg) & $76.4(10.4)$ & $78.8(10.3)$ & $<0.001$ & $82.8(11.1)$ \\
\hline Total cholesterol $\left(\mathrm{mg} \mathrm{dl}^{-1}\right)$ & $195.7(31.2)$ & $197.9(29.8)$ & 0.106 & $252.1(31.1)$ \\
\hline Triglyceride $\left(\mathrm{mg} \mathrm{dl}^{-1}\right)$ & $108.5(72.3)$ & $131.9(108.7)$ & $<0.001$ & $155.9(69.1)$ \\
\hline HDL-C (mg dl $\left.{ }^{-1}\right)$ & $53.4(13.8)$ & $48.3(12.1)$ & $<0.001$ & $47.4(12.3)$ \\
\hline LDL-C $\left(\mathrm{mg} \mathrm{dl}^{-1}\right)$ & $117.7(27.1)$ & $121.1(25.7)$ & $<0.001$ & $170.6(25.6)$ \\
\hline HOMA-IR & $1.5(1.3)$ & $2.4(2.0)$ & $<0.001$ & $2.9(3.2)$ \\
\hline Metabolic syndrome $^{\mathrm{b}}(\%)$ & 7.7 & 22.0 & & \\
\hline
\end{tabular}

Abbreviations: BP, blood pressure; CRP, C-reactive protein; HDL-C, high-density lipoprotein cholesterol; HOMA-IR, insulin resistance based on homeostasis model assessment; LDL-C, low-density lipoprotein cholesterol. Participants who were not eligible for statin therapy were further divided into low CRP and high CRP group. ${ }^{*} P$-value (two-sided) comparing subgroup with CRP $\leqslant 2 \mathrm{mgl}^{-1}$ versus CRP $>2 \mathrm{mgl}^{-1}$. Data are expressed as mean (standard deviation) or $\%$ (proportion of the

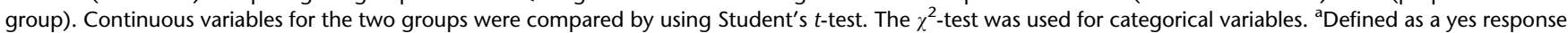
to the questions, 'Have you ever smoked cigarettes?' and 'Do you smoke now?' betabolic syndrome as defined by the International Diabetes Federation. 
Of those with elevated CRP, a greater proportion had three or more risk factors compared with those with normal CRP. The prevalence of metabolic syndrome was higher in participants with CRP $>2 \mathrm{mgl}^{-1}$ when compared with those with CRP $\leqslant 2 \mathrm{mgl}^{-1}$ (22.0 versus $7.7 \%, P<0.001$ ).

We next performed multivariable logistic regression analysis to identify independent predictors of elevated CRP levels in participants who were not eligible for statin therapy (Table 2). Compared with univariate models, only gender (female, OR 3.34 95\% CI 2.68-4.16), ethnicity (Malays, OR 1.57 95\% CI 1.25-1.96; Asian Indians OR 1.97, 95\% CI 1.55-2.50), waist circumference (OR 1.06 95\% CI 1.05-1.07) and IR (OR 1.14 95\% CI 1.07-1.22) were statistically significant (all $P$-values $<0.001$ ), although the effect of ethnicity, waist circumference and IR appeared attenuated compared with the univariate analyses. Interestingly, cigarette smoking became a significant predictor of elevated CRP levels after adjustment for other factors (OR 1.49, 95\% CI 1.08-2.05, $P=0.011)$.

\section{Discussion}

In this study, we observed that $12.6 \%$ of our population qualified for statin therapy, based on the risk of CHD events estimated using the FRS and their LDL-C levels. Of those who did not qualify for statin therapy, approximately one-third had elevated CRP levels (defined as $>2 \mathrm{mgl}^{-1}$ ). These individuals were equally distributed across the various FRS risk categories and some, particularly those in the intermediate risk group, may benefit from aggressive CHD risk reduction therapy, including a statin therapy. ${ }^{19}$ The findings from the recently published JUPITER study provided evidence that statin therapy in these individuals with low LDL-C levels but elevated CRP conferred a comparable degree of risk reduction and similar number needed to treat as other widely accepted interventions for the primary prevention of cardiovascular disease. ${ }^{20}$

We also found that the features of the metabolic syndrome were more common in the participants with high CRP levels when compared with those with low CRP levels. This is in line with other studies that have shown that the metabolic syndrome is associated with elevated CRP. ${ }^{21-23}$ Following multivariate analysis, we found two independent risk factors for elevated CRP levels that are modifiable, that is, smoking and elevated waist circumference. Again, this is in line with a previous study showing that subjects with metabolic syndrome had higher levels of CRP and the main determinant of the CRP elevation was waist circumference. ${ }^{24} \mathrm{~A}$ recent systematic review suggests that weight loss, regardless of whether it occurs through surgical, lifestyle, dietary and/or exercise intervention, is an effective strategy for lowering CRP levels. ${ }^{25}$ Other studies have also shown that CRP levels are elevated among smokers and decreased with long-term smoking cessation. ${ }^{26,27}$ Weight loss and smoking cessation are also effective in reducing IR, and may be particularly important interventions among those classified as low CHD risk (i.e., 0 to 1 risk factor or Framingham risk estimate of $<10 \%$ ), in whom the evidence for statin therapy is limited. ${ }^{28,29}$

In this multi-ethnic population, we also found that CRP levels varied substantially between individuals of different ethnic groups. When compared with Chinese, elevated CRP $\left(>2 \mathrm{mg} \mathrm{l}^{-1}\right.$ ) levels were about two times more likely in Asian Indians and Malays, even after adjustment for other known variables that might affect CRP levels. This is consistent with

Table 2 Determinants of CRP levels in participants who were not eligible for statin therapy, based on the NCEP-ATPIII guideline

\begin{tabular}{|c|c|c|c|c|}
\hline & Unadjusted OR & P-value & Multivariate $O R$ & P-value \\
\hline \multicolumn{5}{|l|}{ Gender } \\
\hline Male & 1.00 & - & 1.00 & - \\
\hline Female & $1.45(1.23,1.70)$ & $<0.001$ & $3.34(2.68,4.16)$ & $<0.001$ \\
\hline \multicolumn{5}{|l|}{ Race } \\
\hline Chinese & 1.00 & - & 1.00 & - \\
\hline Malays & $2.37(1.94,2.90)$ & $<0.001$ & $1.57(1.25,1.96)$ & $<0.001$ \\
\hline Asian Indians & $3.67(2.98,4.53)$ & $<0.001$ & $1.97(1.55,2.50)$ & $<0.001$ \\
\hline Current smoking & $0.92(0.70,1.22)$ & 0.577 & $1.49(1.08,2.05)$ & 0.011 \\
\hline History of hypertension & $1.81(1.47,2.22)$ & $<0.001$ & $1.11(0.85,1.43)$ & 0.236 \\
\hline Age & $1.02(1.01,1.03)$ & $<0.001$ & $1.00(0.99,1.01)$ & 0.381 \\
\hline Waist circumference & $1.07(1.06,1.08)$ & $<0.001$ & $1.06(1.05,1.08)$ & $<0.001$ \\
\hline Systolic BP & $1.02(1.01,1.02)$ & $<0.001$ & $1.00(1.00,1.01)$ & 0.396 \\
\hline Total cholesterol & $1.11(1.00,1.23)$ & 0.036 & $0.73(0.30,1.76)$ & 0.595 \\
\hline Triglyceride & $1.004(1.003,1.005)$ & $<0.001$ & $1.00(1.00,1.01)$ & 0.735 \\
\hline HDL-C & $0.97(0.96,0.98)$ & $<0.001$ & $0.99(0.97,1.01)$ & 0.390 \\
\hline LDL-C & $1.005(1.002,1.008)$ & 0.001 & $1.01(0.99,1.04)$ & 0.411 \\
\hline HOMA-IR & $1.50(1.41,1.59)$ & $<0.001$ & $1.14(1.07,1.22)$ & $<0.001$ \\
\hline
\end{tabular}

Abbreviations: BP, blood pressure; CRP, C-reactive protein; FPG, fasting plasma glucose; HDL-C, high-density lipoprotein cholesterol; HOMA-IR, insulin resistance based on homeostasis model assessment; LDL-C, low-density lipoprotein cholesterol; OR, odds ratio. Data expressed as OR (95\% confidence interval). Bold values are those with $P$-value $<0.05$. 
our previous findings that Asian Indians have greater risk of CHD compared with Malays or Chinese. ${ }^{30}$ However, whether elevated CRP levels have a direct causal relationship with atherosclerosis or merely reflect a greater burden of atherosclerosis in the Asian Indians is yet to be elucidated. Such ethnic differences were also observed in the Study of Women's Health Across the Nation (SWAN) cohort, which found that CRP levels varied significantly between ethnic groups, with the highest concentrations seen in AfricanAmerican participants, followed in order by Hispanic, White, Chinese and Japanese participants. ${ }^{31}$ Despite the lower CRP levels in Chinese, we still observed that a substantial proportion had CRP $>2 \mathrm{mg} \mathrm{dl}^{-1}$.

In addition to ethnic differences, female gender is a strong predictor of CRP levels in this population. This finding resonates with findings from several other studies, including the Multi-ethnic study of Atherosclerosis that demonstrated a higher CRP levels in women compared with men, even after adjusting for body mass index and other common confounding variables. ${ }^{32,33}$ However, several studies did not show the gender differences in CRP levels. ${ }^{34,35}$ Recently, in a large population based-sample with accurate adipose tissue quantification by dual-energy X-ray absorptiometry and abdominal magnetic resonance imaging, Khera et al. ${ }^{36}$ reported that higher CRP levels in women compared with men might be explained by the differences in the amount of total fat mass and regional distribution of fat. At this time, the reasons for higher CRP levels in women remain unclear.

Strengths of the present study include data collected from a large sample of community-based individuals. The population also consists of three major ethnic groups residing in Singapore, which represent several of the largest ethnic groups in Asia where the burden of cardiovascular disease is increasing most rapidly. ${ }^{37}$ In addition, a comprehensive assessment of CHD risk factors was available, including an assessment of peripheral arterial disease. This study has limitations worth mentioning. Firstly, the cross-sectional nature of our study does not allow us to estimate the long-term impact of elevated CRP levels on the risk of CHD after the traditional cardiovascular risk factors are taken into account. Secondly, we measured CRP on a single occasion. This could lead to some misclassification. However, the proportions of individuals with elevated CRP level are sufficiently high $(\sim 32 \%)$ that even if this were an overestimate, it would remain a significant problem in the population. Furthermore, we anticipate that any measurement error would be non-differential in relation to the risk factors identified and would therefore bias our findings toward the null hypothesis of no association. Thus, we do not feel that any misclassification would significantly alter our conclusions.

In summary, in a multi-ethnic Asian population living in Singapore, elevated CRP level affects a significant proportion of individuals who would not qualify for statin therapy based on the LDL-C levels. This was more common in women and in those of Malay and Asian-Indian ethnicity. Whether extending statin therapy to this segment of population to reduce incidence of $\mathrm{CHD}$ is not yet clear. Even if drug therapy is not recommended, this presence of elevated CRP should motivate the physician and patient to reinforce healthy lifestyle, targeted at reducing obesity and smoking cessation to reduce the proportion of individuals with elevated CRP levels. These lifestyle interventions should be integrated in any preventive strategy to reduce the burden of CHD and may be particularly pertinent to those at low risk of CHD. Finally, the findings of ethnic and gender differences in CRP levels merit further investigations and may shed light on the pathogenesis of atherosclerosis.

\section{Conflict of interest}

Dr E Shyong Tai has served on advisory boards for Merck Sharp and Dohme (IA Corp), Astra Zeneca and Novo Nordisk. $\mathrm{He}$ is also the principle investigator of an investigator initiated research grant funded by Pfizer, which seeks to identify biological, lifestyle and psychosocial factors associated with individual trajectories of cardiovascular risk factors in South East Asia.

\section{Author contributions}

Dr E Shyong Tai and Dr Jeannette Lee helped in the conception and design the study. Dr Chin Meng Khoo, Maudrene Tan and $\mathrm{Wu}$ Yi helped conduct the literature review, data analysis and preparation of the text. Dr Daniel Wai and Dr Subramaniam Tavintharan helped in the final approval of the manuscript.

\section{References}

1 Wilson PW, D'Agostino RB, Levy D, Belanger AM, Silbershatz H, Kannel WB. Prediction of coronary heart disease using risk factor categories. Circulation 1998; 97: 1837-1847.

2 Ross R. Atherosclerosis - an inflammatory disease. $N$ Engl J Med 1999; 340: 115-126.

3 Libby P. Inflammation in atherosclerosis. Nature 2002; 420: 868-874.

4 Ridker PM, Paynter NP, Rifai N, Gaziano JM, Cook NR. C-reactive protein and parental history improve global cardiovascular risk prediction: the Reynolds Risk Score for men. Circulation 2008; 118: 2243-2251, 4p following 2251.

5 Ridker PM, Buring JE, Rifai N, Cook NR. Development and validation of improved algorithms for the assessment of global cardiovascular risk in women: the Reynolds Risk Score. JAMA 2007; 297: 611-619.

6 Ridker PM, Danielson E, Fonseca FA, Genest J, Gotto Jr AM, Kastelein JJ et al. Rosuvastatin to prevent vascular events in men and women with elevated C-reactive protein. N Engl J Med 2008; 359: 2195-2207.

7 Lee YJ, Lee JH, Shin YH, Kim JK, Lee HR, Lee DC. Gender difference and determinants of C-reactive protein level in Korean adults. Clin Chem Lab Med 2009; 47: 863-869.

8 Matsushita K, Yatsuya H, Tamakoshi K, Yang PO, Otsuka R, Wada $\mathrm{K}$ et al. High-sensitivity C-reactive protein is quite low in Japanese men at high coronary risk. Circ J 2007; 71: 820-825. 
9 Wen J, Liang Y, Wang F, Sun L, Guo Y, Duan X et al. Association of C-reactive protein and metabolic syndrome in a rural Chinese population. Clin Biochem 2009; 42: 976-983.

10 Ye X, Yu Z, Li H, Franco OH, Liu Y, Lin X. Distributions of $\mathrm{C}$-reactive protein and its association with metabolic syndrome in middle-aged and older Chinese people. J Am Coll Cardiol 2007; 49: 1798-1805.

11 Iso H, Cui R, Date C, Kikuchi S, Tamakoshi A. C-reactive protein levels and risk of mortality from cardiovascular disease in Japanese: the JACC Study. Atherosclerosis 2009; 207: 291-297.

12 Hughes K, Yeo PP, Lun KC, Thai AC, Sothy SP, Wang KW et al. Cardiovascular diseases in Chinese, Malays, and Indians in Singapore. II. Differences in risk factor levels. J Epidemiol Community Health 1990; 44: 29-35.

13 Tan CE, Emmanuel SC, Tan BY, Jacob E. Prevalence of diabetes and ethnic differences in cardiovascular risk factors. The 1992 Singapore National Health Survey. Diabetes Care 1999; 22: 241-247.

14 Hughes K, Aw TC, Kuperan P, Choo M. Central obesity, insulin resistance, syndrome $\mathrm{X}$, lipoprotein(a), and cardiovascular risk in Indians, Malays, and Chinese in Singapore. J Epidemiol Community Health 1997; 51: 394-399.

15 Cutter J, Tan BY, Chew SK. Levels of cardiovascular disease risk factors in Singapore following a national intervention programme. Bull World Health Organ 2001; 79: 908-915.

16 Jiang S, Bao Y, Hou X, Fang Q, Wang C, Pan J et al. Serum C-reactive protein and risk of cardiovascular events in middle-aged and older Chinese population. Am I Cardiol 2009; 103: 1727-1731.

17 Alberti KG, Zimmet P, Shaw J. Metabolic syndrome - a new worldwide definition. A consensus statement from the International Diabetes Federation. Diabet Med 2006; 23: 469-480.

18 National Cholesterol Education Program (NCEP) Expert Panel on Detection, Evaluation, and Treatment of High Blood Cholesterol in Adults (Adult Treatment Panel III). Third Report of the National Cholesterol Education Program (NCEP) Expert Panel on Detection, Evaluation, and Treatment of High Blood Cholesterol in Adults (Adult Treatment Panel III) final report. Circulation 2002; 106: 3143-3421.

19 Pearson TA, Mensah GA, Alexander RW, Anderson JL, Cannon III RO, Criqui $\mathrm{M}$ et al. Markers of inflammation and cardiovascular disease: application to clinical and public health practice: a statement for healthcare professionals from the Centers for Disease Control and Prevention and the American Heart Association. Circulation 2003; 107: 499-511.

20 Ridker PM, MacFadyen JG, Fonseca FA, Genest J, Gotto AM, Kastelein JJ et al. Number needed to treat with rosuvastatin to prevent first cardiovascular events and death among men and women with low low-density lipoprotein cholesterol and elevated high-sensitivity C-reactive protein: justification for the use of statins in prevention: an intervention trial evaluating rosuvastatin (JUPITER). Circ Cardiovasc Qual Outcomes 2009; 2: 616-623.

21 Conen D, Rexrode KM, Creager MA, Ridker PM, Pradhan AD. Metabolic syndrome, inflammation, and risk of symptomatic peripheral artery disease in women: a prospective study. Circulation 2009; 120: 1041-1047.

22 Gonzalez AS, Guerrero DB, Soto MB, Diaz SP, Martinez-Olmos M, Vidal O. Metabolic syndrome, insulin resistance and the inflammation markers C-reactive protein and ferritin. Eur J Clin Nutr 2006; 60: 802-809.

23 Ridker PM, Buring JE, Cook NR, Rifai N. C-reactive protein, the metabolic syndrome, and risk of incident cardiovascular events:

an 8-year follow-up of 14719 initially healthy American women. Circulation 2003; 107: 391-397.

24 Nakamura H, Ito H, Egami Y, Kaji Y, Maruyama T, Koike G et al. Waist circumference is the main determinant of elevated C-reactive protein in metabolic syndrome. Diabetes Res Clin Pract 2008; 79: 330-336.

25 Selvin E, Paynter NP, Erlinger TP. The effect of weight loss on C-reactive protein: a systematic review. Arch Intern Med 2007; 167: 31-39.

26 Bazzano LA, He J, Muntner P, Vupputuri S, Whelton PK. Relationship between cigarette smoking and novel risk factors for cardiovascular disease in the United States. Ann Intern Med 2003; 138: 891-897.

27 Wannamethee SG, Lowe GD, Shaper AG, Rumley A, Lennon L, Whincup PH. Associations between cigarette smoking, pipe/cigar smoking, and smoking cessation, and haemostatic and inflammatory markers for cardiovascular disease. Eur Heart J 2005; 26: 1765-1773.

28 Eliasson B, Attvall S, Taskinen MR, Smith U. Smoking cessation improves insulin sensitivity in healthy middle-aged men. Eur J Clin Invest 1997; 27: 450-456.

29 Gerich JE. Contributions of insulin-resistance and insulinsecretory defects to the pathogenesis of type 2 diabetes mellitus. Mayo Clin Proc 2003; 78: 447-456.

30 Ounpuu S, Yusuf S. Singapore and coronary heart disease: a population laboratory to explore ethnic variations in the epidemiologic transition. Eur Heart I 2003; 24: 127-129.

31 Matthews KA, Sowers MF, Derby CA, Stein E, Miracle-McMahill H, Crawford SL et al. Ethnic differences in cardiovascular risk factor burden among middle-aged women: Study of Women's Health Across the Nation (SWAN). Am Heart J 2005; 149: 1066-1073.

32 Lakoski SG, Cushman M, Criqui M, Rundek T, Blumenthal RS, D'Agostino Jr RB et al. Gender and C-reactive protein: data from the Multiethnic Study of Atherosclerosis (MESA) cohort. Am Heart J 2006; 152: 593-598.

33 Khera A, McGuire DK, Murphy SA, Stanek HG, Das SR, Vongpatanasin $\mathrm{W}$ et al. Race and gender differences in C-reactive protein levels. J Am Coll Cardiol 2005; 46: 464-469.

34 Rifai N, Ridker PM. Population distributions of C-reactive protein in apparently healthy men and women in the United States: implication for clinical interpretation. Clin Chem 2003; 49: 666-669.

35 Hutchinson WL, Koenig W, Frohlich M, Sund M, Lowe GD, Pepys MB. Immunoradiometric assay of circulating C-reactive protein: age-related values in the adult general population. Clin Chem 2000; 46: 934-938.

36 Khera A, Vega GL, Das SR, Ayers C, McGuire DK, Grundy SM et al. Sex differences in the relationship between C-reactive protein and body fat. J Clin Endocrinol Metab 2009; 94: 3251-3258.

37 Yusuf S, Reddy S, Ounpuu S, Anand S. Global burden of cardiovascular diseases: part I: general considerations, the epidemiologic transition, risk factors, and impact of urbanization. Circulation 2001; 104: 2746-2753.

(c)

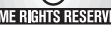

This work is licensed under the Creative Commons Attribution-NonCommercial-No Derivative Works 3.0 Unported License. To view a copy of this license, visit http://creativecommons.org/ licenses/by-nc-nd/3.0/ 